OPEN ACCESS

Edited by:

Joshua Gordon,

Columbia University, USA

Reviewed by:

Vikaas Singh Sohal,

University of California,

San Francisco, USA

Avishek Adhikari,

Stanford University, USA

Eero Castrén,

University of Helsinki, Finland

*Correspondence:

Matthias Eder

eder@psych.mpg.de

Received: 22 August 2015 Accepted: 22 October 2015

Published: 06 November 2015

Citation:

Stepan J, Hladky F, Uribe A, Holsboer F, Schmidt MV and Eder M (2015)

High-Speed imaging reveals opposing effects of chronic stress and antidepressants on neuronal activity propagation through the hippocampal trisynaptic circuit.

Front. Neural Circuits 9:70. doi: 10.3389/fncir.2015.00070

\section{High-Speed imaging reveals opposing effects of chronic stress and antidepressants on neuronal activity propagation through the hippocampal trisynaptic circuit}

\author{
Jens Stepan 1,2,3,4, Florian Hladky 1,2,3, Andrés Uribe ${ }^{1,2,5}$, Florian Holsboer ${ }^{1,6}$, \\ Mathias V. Schmidt ${ }^{1,2,5}$ and Matthias Eder ${ }^{1,2,3 *}$
}

${ }^{1}$ Max Planck Institute of Psychiatry, Munich, Germany, ${ }^{2}$ Department Stress Neurobiology and Neurogenetics, Max Planck Institute of Psychiatry, Munich, Germany, ${ }^{3}$ Scientific Core Unit "Electrophysiology and Neuronal Network Dynamics", Max Planck Institute of Psychiatry, Munich, Germany, ${ }^{4}$ Clinical Department, Max Planck Institute of Psychiatry, Munich, Germany, ${ }^{5}$ Research Group "Stress Resilience", Max Planck Institute of Psychiatry, Munich, Germany, ${ }^{6}$ HMNC GmbH, Munich, Germany

Antidepressants (ADs) are used as first-line treatment for most stress-related psychiatric disorders. The alterations in brain circuit dynamics that can arise from stress exposure and underlie therapeutic actions of ADs remain, however, poorly understood. Here, enabled by a recently developed voltage-sensitive dye imaging (VSDI) assay in mouse brain slices, we examined the impact of chronic stress and concentration-dependent effects of eight clinically used ADs (belonging to different chemical/functional classes) on evoked neuronal activity propagations through the hippocampal trisynaptic circuitry (HTC: perforant path $\rightarrow$ dentate gyrus (DG) $\rightarrow$ area CA3 $\rightarrow$ area CA1). Exposure of mice to chronic social defeat stress led to markedly weakened activity propagations ("HTC-Waves"). In contrast, at concentrations in the low micromolar range, all ADs, which were bath applied to slices, caused an amplification of HTC-Waves in CA regions (invariably in area CA1). The fast-acting "antidepressant" ketamine, the mood stabilizer lithium, and brain-derived neurotrophic factor (BDNF) exerted comparable enhancing effects, whereas the antipsychotic haloperidol and the anxiolytic diazepam attenuated HTC-Waves. Collectively, we provide direct experimental evidence that chronic stress can depress neuronal signal flow through the HTC and demonstrate shared opposing effects of ADs. Thus, our study points to a circuit-level mechanism of ADs to counteract stress-induced impairment of hippocampal network function. However, the observed effects of ADs are impossible to depend on enhanced neurogenesis.

Keywords: hippocampus, trisynaptic circuit, activity propagation, stress, antidepressants, ketamine, voltagesensitive dye, imaging 


\section{INTRODUCTION}

Stress is a major environmental risk factor for the development of several psychiatric diseases, including depression and anxiety disorders (de Kloet et al., 2005; Krishnan and Nestler, 2008; Popoli et al., 2012). To date, the first-line treatment for most stress-related psychiatric disorders is administration of antidepressants (ADs; Benkert and Hippius, 2012). While intensive research has provided fundamental insights into stressinduced brain abnormalities and mechanisms of $\mathrm{AD}$ action at the molecular, cellular, and synaptic level (Castrén, 2005; de Kloet et al., 2005; Krishnan and Nestler, 2008; Popoli et al., 2012; Hill et al., 2015), the alterations in millisecond-scale neuronal circuit dynamics that can arise from stress exposure and underlie therapeutic actions of ADs are largely unknown. Investigations on this topic appear crucial, since many symptoms of psychiatric disorders and their pharmacological alleviation are increasingly thought to be best reflected by changes in spatiotemporal patterns of electrical neuronal activity (Castrén, 2005; Airan et al., 2007; Karayiorgou et al., 2012; Monteggia et al., 2014).

A brain structure that is highly sensitive to stress hormones, involved in the regulation of stress responses, and most likely a therapeutically relevant target of ADs is the hippocampus (Andersen et al., 2007; Maggio and Segal, 2007; von Wolff et al., 2011; Méndez et al., 2012; Hill et al., 2015). Stress can severely affect the structural integrity of the hippocampal network and it is widely accepted that at least a part of the resultant neurophysiological changes contribute to the symptomatology of depression and other stress-related psychiatric diseases (de Kloet et al., 2005; Popoli et al., 2012). A major function of the hippocampus is to process sensory information, to store it if appropriate, and to transmit it to downstream brain structures. Transfer of sensory information to the hippocampus prominently takes place via perforant path fibers (originating in entorhinal cortex layer II) that synapse on neurons of the dentate gyrus (DG). The DG represents the first relay station of the glutamatergic trisynaptic circuitry of the hippocampus (in the following abbreviated HTC: perforant path $\rightarrow$ DG $\rightarrow$ area CA3 $\rightarrow$ CA1 output subfield, Figure 1A; Andersen et al., 2007).

We recently developed a voltage-sensitive dye imaging (VSDI) assay in mouse brain slices, allowing real-time monitoring and, thus, the investigation of neuronal activity propagations through the entire HTC network ("HTC-Waves"; Stepan et al., 2012, 2015). In this assay (Figure 1), HTC-Waves are triggered by theta-frequency stimulation of perforant path fibers, mimicking synchronous theta-rhythmical spiking of entorhinal layer II neurons, which exhibit such activity during physiologically highly relevant theta oscillations in the entorhinal-hippocampal system (Dickson et al., 2000; Mizuseki et al., 2009; Quilichini et al., 2010; Burgalossi et al., 2011). Here, by use of the HTC-Wave assay, we are the first to examine the impact of chronic stress and concentration-dependent effects of $\mathrm{ADs}$ on polysynaptic activity propagations through a major input-output network of the hippocampus.

VSDI in brain slices has been already previously employed to investigate stress and $\mathrm{AD}$ effects on hippocampal circuit

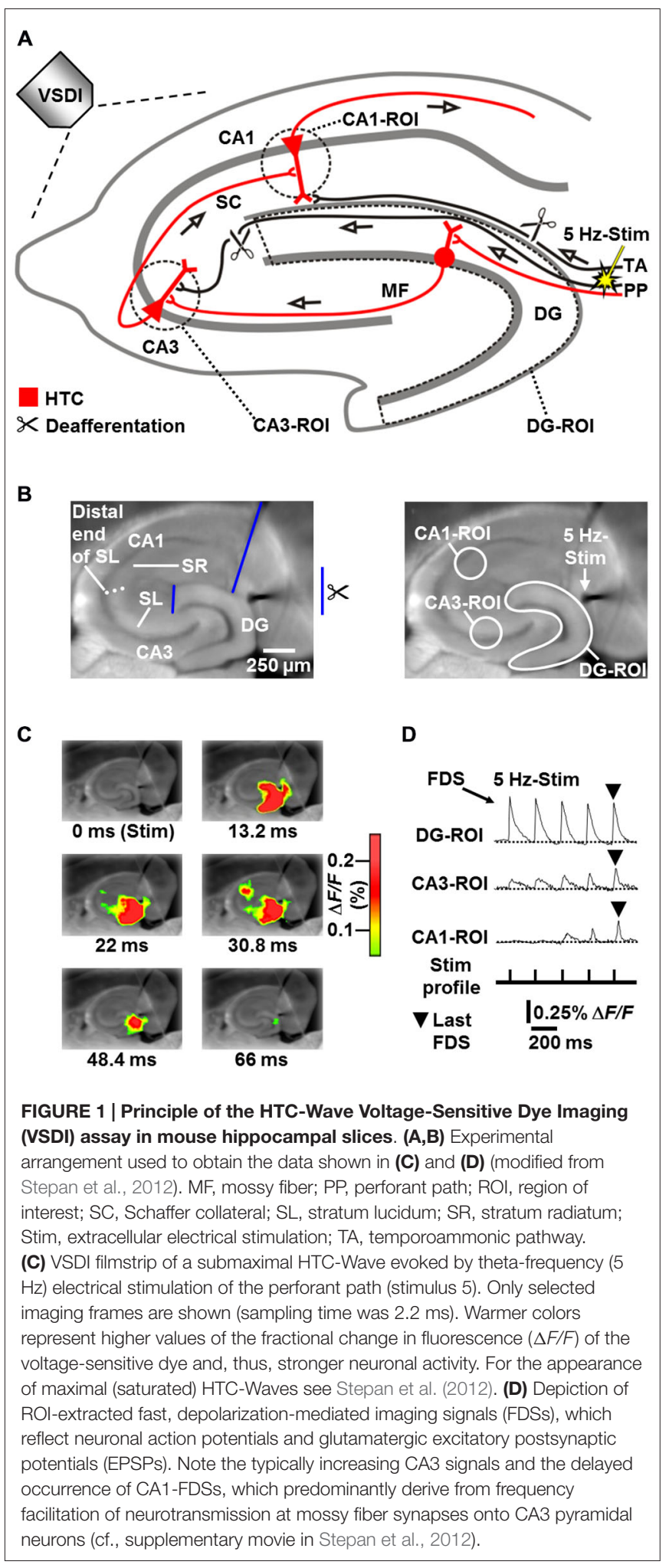

dynamics (Airan et al., 2007). In this work, rats were exposed to chronic mild stress or treated with fluoxetine or imipramine for several days. The authors found that a measure of local activity spread within the DG was 
decreased in slices from stressed animals and observed an opposing effect in area CA1. Antipodal neurophysiological changes were detected in slices from AD-treated animals. In contrast to the present study, neuronal activity in the DG and area CA1 was evoked independently from each other by local electrical stimulation, thus excluding measurements of activity propagation through the whole HTC network and potential effects on the highly stress-sensitive CA3 circuitry.

To summarize our main results, we found that exposure of mice to chronic social defeat stress leads to markedly weakened HTC-Waves, while all eight ADs tested (belonging to different chemical/functional classes) amplify the activity propagations in CA regions at concentrations in the low micromolar range.

\section{MATERIALS AND METHODS}

\section{Animals}

For all experiments, adult male $\mathrm{C} 57 \mathrm{BL} / 6 \mathrm{~N}$ mice (8 to 12 weeks old, obtained from the Max Planck Institute's breeding colony) and adult male CD-1 mice (12 to 24 weeks old, purchased from Charles River) were used. All experimental procedures were approved by the committee for the Care and Use of Laboratory animals of the Government of Upper Bavaria, Germany.

\section{Chronic Social Defeat Stress}

The stress paradigm lasted 14-19 days and was conducted as we described previously (Wagner et al., 2012). Briefly, the experimental $\mathrm{C} 57 \mathrm{BL} / 6 \mathrm{~N}$ mice were introduced into the home cage of a dominant resident CD-1 mouse and defeated shortly after. When the defeat was achieved, the animals were separated by a wire mesh, preventing physical but allowing sensory contact for $24 \mathrm{~h}$. Each day, animals of the stress group were defeated by another unfamiliar, dominant resident mouse, excluding a repeated encounter throughout the experiment. The daily defeat was performed between 11:00 am and 4:00 pm. Varying starting times reduced the predictability of the stressor and, therefore, minimized a potential habituation effect. Control mice were housed in their home cages during the course of the experiment. Both stressed and control animals were handled daily during the stress procedure. At the day of VSDI experimentation, animals of the stress group were not anymore defeated. VSDI measurements were done between day 15 and 20 after the start of the stress paradigm.

\section{Preparation and Staining of Brain Slices}

C57BL/6N mice were anesthetized with isoflurane and decapitated. All following steps were done in ice-cold sucrosebased saline saturated with carbogen gas $\left(95 \% \mathrm{O}_{2} / 5 \% \mathrm{CO}_{2}\right)$. This saline ( $\mathrm{pH} 7.4$ ) consisted of (in $\mathrm{mM}): 87 \mathrm{NaCl}, 2.5$ $\mathrm{KCl}, 25 \mathrm{NaHCO}_{3}, 1.25 \mathrm{NaH}_{2} \mathrm{PO}_{4}, 0.5 \mathrm{CaCl}_{2}, 7 \mathrm{MgCl}_{2}, 25$ glucose, and 75 sucrose. The hemispheres were prepared for the slicing procedure by a special transversal cut, which is sometimes called "magic cut" (Bischofberger et al., 2006). We cut the hemispheres at angles optimized to conserve the intrahippocampal axonal projections along the DG$\mathrm{CA}$ axis as best as possible. Subsequently, $350 \mu \mathrm{m}$-thick horizontal slices were cut using a vibratome (HM650V, Thermo Scientific). Slices were incubated in carbogenated sucrose-based saline for $30 \mathrm{~min}$ at $34^{\circ} \mathrm{C}$. Subsequent staining with the voltage-sensitive dye Di-4-ANEPPS (dissolved in DMSO to a $20.8 \mathrm{mM}$ stock solution) was carried out at room temperature $\left(23-25^{\circ} \mathrm{C}\right)$. Slices were kept for $15 \mathrm{~min}$ in carbogenated physiological saline containing Di-4-ANEPPS (7.5 $\mu \mathrm{g} / \mathrm{ml},<0.1 \%$ DMSO). The physiological saline ( $\mathrm{pH} 7.4$ ) consisted of (in mM): $125 \mathrm{NaCl}, 2.5 \mathrm{KCl}, 25 \mathrm{NaHCO}_{3}, 1.25$ $\mathrm{NaH}_{2} \mathrm{PO}_{4}, 2 \mathrm{CaCl}_{2}, 1 \mathrm{MgCl}_{2}$, and 25 glucose. Afterwards, slices were stored at room temperature for at least $30 \mathrm{~min}$ in Di-4-ANEPPS-free carbogenated physiological saline containing $0.6 \mu \mathrm{M}$ bicuculline methiodide (BIM, for rationale see "Discussion" Section).

\section{VSDI}

VSDI and data analysis were performed using the MiCAM02 hard and software package (BrainVision). The tandem-lens fluorescence microscope was equipped with the MiCAM02-HR camera and the $2 \times$ and $1 \times$ lens at the objective and condensing side, respectively (for further technical details see http://www.scimedia.com). Acquisition settings were as follows: $88 \times 60$ pixels frame size, $36.4 \times 40.0 \mu \mathrm{m}$ pixel size, and $2.2 \mathrm{~ms}$ sampling time.

\section{Processing and Quantification of VSDI Data}

From VSDI signals, the fractional change in fluorescence $(\Delta F / F)$ was calculated and $\Delta F / F$ values were spatially and temporally smoothed using a $3 \times 3 \times 3$ average filter. VSDI signals presented in images were smoothed with a $5 \times 5 \times 3$ average filter. To attenuate slow signal components produced from bleaching of the dye and slight summation of $5 \mathrm{~Hz}$ neuronal responses (see next paragraph), we afterwards applied a weak high-pass filter of the MiCAM02 software $(\tau=220 \mathrm{~ms})$ to the imaging data. Pixelation of images was reduced by the interpolation function of the MiCAM02 software.

For analysis of neuronal population activity in hippocampal subregions, standardized regions of interest (ROIs) were manually set according to anatomical landmarks. The circular CA3-ROI ( $r=4$ pixels) was positioned into the CA3 region near the DG, but not overlapping with it. The circular CA1-ROI $(r=4$ pixels $)$ was placed into the CA1 subfield with a distance of approximately 200 $\mu \mathrm{m}$ from the visually identified distal end of the stratum lucidum. Both ROIs spanned the stratum oriens, stratum pyramidale, and stratum lucidum/radiatum. The DG-ROI, which enclosed the fascia dentata (Figures 1A,B), was created by the polygon-drawing function of the MiCAM02 software. The average of smoothed $\Delta F / F$ values within a particular ROI served as final measure of neuronal population activity. 


\section{Brain Slice Experiments}

All slice experiments were carried out at room temperature and slices were continuously superfused with BIM $(0.6 \mu \mathrm{M})$ containing carbogenated physiological saline $(4-5 \mathrm{ml} / \mathrm{min}$ flow rate). BIM never led to epileptiform activity in the hippocampal subfields under study (for the appearance of such activity see Stepan et al., 2012). HTC-Waves were evoked by square pulse electrical stimuli (200 $\mu$ s pulse width) delivered at $5 \mathrm{~Hz}$ via a monopolar tungsten electrode (50 $\mu \mathrm{m}$ tip diameter, $\sim 0.5 \mathrm{M} \Omega$ nominal impedance) to the visually identified perforant path near its entry zone to the DG (Figures 1A,B). In all slices, perforant path fibers which directly innervate CA3 pyramidal cells were cut at the point where they exit the DG. Temporoammonic projections were likewise functionally inactivated (Figures 1A,B). For the pharmacological experiments, the intensity of perforant path stimulation $(15-35 \mathrm{~V})$ was adjusted in a manner to produce fast, depolarization-mediated imaging signals (FDSs) in the DG with amplitudes of $50-80 \%$ of the highest attainable value. These FDSs range within the linear upturn of the respective input-output curve (Stepan et al., 2012). To obtain submaximal HTC-Waves comprising CA1-FDSs with amplitudes of $0.1-0.3 \%$ $\Delta F / F, 4-7$ consecutive stimulation pulses were delivered per recording sequence to the perforant path (Figures 1C,D). The number of consecutive stimulation pulses was held constant within an experiment. Acquisitions were made every $2 \mathrm{~min}$ and the amplitude values of the last DG-, CA3-, and CA1FDS within a recording sequence (Figure 1D) were determined by means of the MiCAM02 software. As final measure of DG, CA3, and CA1 activity, we calculated mean DG-, CA3-, and CA1-FDS amplitude values over three consecutive acquisitions. Drugs were bath applied to slices if baseline recording was stable over $20 \mathrm{~min}$ (Figure 3A). For each pharmacological condition, a maximum number of two slices per animal was used.

For the experiments in slices from stressed/non-stressed mice, we used a HTC-Wave assay, which was refined for group comparisons. We established an experimental procedure, by which inter-slice variability in HTC dynamics could be well handled. For each animal, this procedure was applied to 2-3 slices. In particular, we delivered three trains of 15 stimulation pulses ( $5 \mathrm{~Hz}$ stimulation, $3 \mathrm{~min}$ inter-train interval) to the perforant path. The stimulation intensity was adjusted such that the first DG-FDS within recording sequence one, two, and three had an amplitude of approximately $0.35,0.45$, and $0.55 \% \Delta F / F$, respectively (Figure 2B). The resultant HTCWaves comprised CA3- and CA1-FDSs, which linearly increased with increasing stimulation intensity (data not shown). For quantification, we used the amplitude value of the first DGFDS, the mean amplitude value of the last three CA3-FDSs, and the mean amplitude value of the last three CA1-FDSs within a recording sequence. These values were averaged over the three recording sequences. The resultant mean values in turn were averaged over the 2-3 experiments conducted for each animal, yielding the measure "Mean FDS amplitude" (Figure 2C). Healthiness of "stress" slices was comparable to that of "control" slices, since the ranges of stimulation intensities

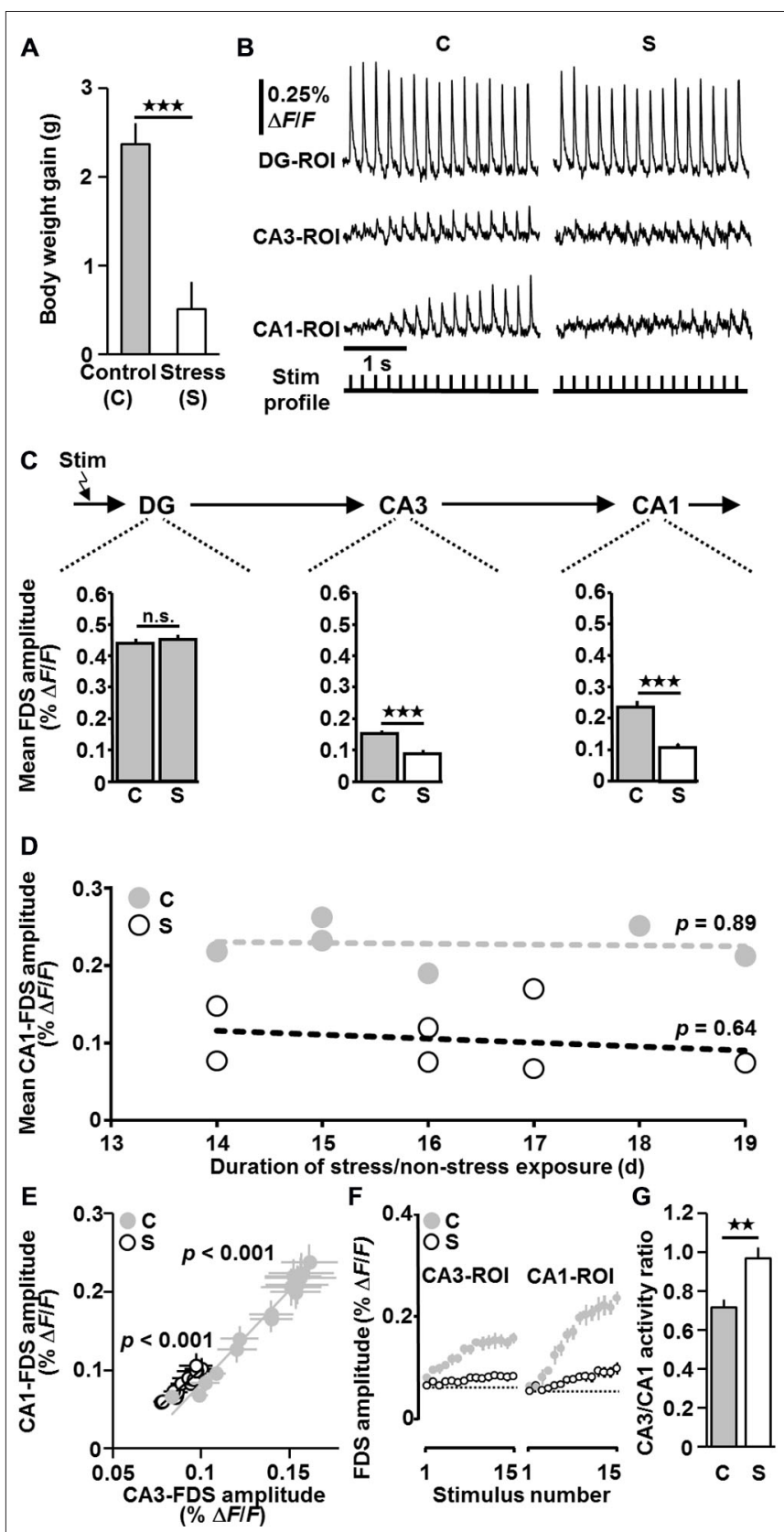

FIGURE 2 | Exposure of mice to chronic social defeat stress causes markedly weakened HTC-Waves. (A) Data from body weight measurements (body weight gain during stress/non-stress exposure; stress $(n=7)$ vs. control $(n=6)$ : $t_{(11)}=-4.6, p<0.001$; two-tailed unpaired $t$-test). (B) Representative VSDI recording traces for control and stress conditions. For both recording sequences, the intensity of perforant path stimulation (15 pulses at $5 \mathrm{~Hz}$ ) was adjusted such that the first DG-FDS had an amplitude of $0.45 \% \Delta F / F$. (C) Quantification of VSDI measurements $(n=6$ animals/18 slices for the control group and $n=7$ animals/19 slices for the stress group; VSDI measures obtained from 2-3 slices per animal were averaged, leading to $n$ values of 6 and 7 for the control and stress group, respectively; stress vs. control: CA3: $t_{(11)}=-5.0, p<0.001$; CA1: $t_{(11)}=-5.7, p<0.001$; two-tailed unpaired $t$-tests). For a detailed description of how the measure "Mean FDS amplitude" was calculated see "Materials and Methods". (D) Relationships between mean CA1-FDS amplitude and duration of stress/non-stress exposure ( $p$ values were determined by linear

(Continued) 


\section{FIGURE 2 | Continued}

regression analyses). (E) In slices from both control and stressed mice, the amplitude of CA1-FDSs linearly increased with the amplitude of CA3-FDSs. Data were obtained by averaging the CA1- and CA3-FDS amplitude values for electrical stimulus $1,2,3, \ldots, 15$ over the $18 / 19$ experiments performed in control/stressed mice, in which the amplitude of the first DG-FDS was adjusted to $\sim 0.45 \% \Delta F / F$. (F) The typical increase in CA3- and CA1-FDSs during $5 \mathrm{~Hz}$ perforant path stimulation is much weaker in slices from stressed animals. (G) CA3/CA1 activity ratios for the experiments conducted in control/stressed mice, in which the amplitude of the first DG-FDS was adjusted to $0.45 \% \Delta F / F$ (stress vs. control: $t_{(11)}=3.5, p=0.005$; two-tailed unpaired $t$-test). ${ }^{* *} p<0.01,{ }^{* * *} p<0.001$; n.s., not statistically significant.

needed for evocation of the defined DG-FDSs were nearly identical (6-32 V for "stress" slices and 7-36 V for "control" slices).

\section{Chemicals}

Amitriptyline hydrochloride, BIM, citalopram hydrobromide, clomipramine hydrochloride, Di-4-ANEPPS, diazepam (dissolved in DMSO to a $100 \mathrm{mM}$ stock solution, $\leq 0.001 \%$ final DMSO), DMSO, fluoxetine hydrochloride, fluvoxamine maleate, haloperidol (dissolved in DMSO to a $100 \mathrm{mM}$ stock solution, $\leq 0.02 \%$ final DMSO), S-(+)-ketamine hydrochloride, $\mathrm{LiCl}$, tranylcypromine hydrochloride, venlafaxine hydrochloride, and all salts for the saline solutions were purchased from SigmaAldrich. ANA-12 and tianeptine sodium salt were from Tocris, brain-derived neurotrophic factor (BDNF) from Biomol, and isoflurane from Abbott.

\section{Statistics}

Statistical analyses were run in SigmaStat (Systat Software), with significance declared at $p<0.05\left({ }^{*} p<0.05\right.$, ${ }^{* *} p<0.01$, ${ }^{* * *} p<0.001$; n.s., not statistically significant). Data are given as mean \pm SEM. Statistical tests are described in the respective paragraphs of the results section and/or figure legends. In the pharmacological experiments where ANOVAs were employed for statistical evaluation, the one-sample $t$-test was used to determine the concentration(s) at which a statistically significant effect took place, given that the ANOVA passed $(p<0.05)$.

\section{RESULTS}

\section{Exposure of Mice to Chronic Social Defeat Stress Causes Markedly Weakened HTC-Waves}

We examined the impact of chronic social defeat stress (14-19 days) on HTC-Waves. To assure "pure" activity propagations through the HTC network, perforant path fibers which directly innervate CA3 pyramidal cells were cut at the point where they exit the DG. Temporoammonic projections were likewise functionally inactivated (Figures 1A,B (scissors)). As a realtime measure of neuronal population activity in the DG and CA areas, we employed the amplitude of fast, depolarizationmediated VSDI signals (FDSs, Figure 1D), which reflect action potentials and glutamatergic excitatory postsynaptic potentials (EPSPs; Airan et al., 2007; von Wolff et al., 2011; Stepan et al.,
2012). For more detailed information about the characteristics and physiological relevance of HTC-Waves see "Discussion" Section and Stepan et al. (2012, 2015). The stress paradigm used reliably induces changes in metabolic and endocrine parameters, which typically occur with chronic stress exposure. Furthermore, it causes an increased stress reactivity and an impairment of hippocampus-dependent cognitive abilities (Wang et al., 2011; Wagner et al., 2012). Body weight measurements confirmed that the mice under investigation were profoundly stressed (Figure 2A; Wang et al., 2011). As evident from a pronounced decrease of CA3- and CA1-FDSs, the stress procedure led to considerably weakened HTC-Waves when compared to control conditions (Figures 2B,C). Linear regression analyses showed that there was no relationship between this effect and the duration of stress exposure (Figure 2D).

Further analyses revealed that in slices from both control and stressed mice the amplitude of CA1-FDSs linearly increased with the amplitude of CA3-FDSs (Figure 2E). However, the typical initial increase in CA3- and CA1-FDSs during $5 \mathrm{~Hz}$ perforant path stimulation, which predominantly derives from frequency facilitation of neurotransmission at mossy fiber synapses onto CA3 pyramidal neurons (Stepan et al., 2012), was much weaker in slices from stressed animals (Figure 2F). We also calculated CA3/CA1 activity ratios for "control" and "stress" slices. These ratios were determined by dividing the mean amplitude value of the last three CA3-FDSs by the mean amplitude value of the last three CA1-FDSs within a recording sequence and by averaging the resultant quotients over the 2-3 experiments conducted for each animal. The CA3/CA1 activity ratio was higher in the stress group (Figure 2G). These findings strongly suggest that the stress effect on HTC-Waves originated from impaired neurotransmission in both area CA3 and area CA1.

\section{ADs Exert Enhancing Effects on HTC-Waves}

In another set of experiments, we investigated potential effects of eight clinically used ADs on HTC-Waves. The ADs were the tricyclics amitriptyline and clomipramine, the selective serotonin reuptake inhibitors fluoxetine, citalopram, and fluvoxamine, the selective serotonin/norepinephrine reuptake inhibitor venlafaxine, the selective serotonin reuptake enhancer tianeptine, and the monoamine oxidase $\mathrm{A}$ and $\mathrm{B}$ inhibitor tranylcypromine (McEwen et al., 2010; Benkert and Hippius, 2012). The ADs were tested at $0.1,0.5,1$, 5, 10, and mostly also at 15 and $20 \mu \mathrm{M}$. As illustrated for fluoxetine $(10 \mu \mathrm{M})$, which significantly increased CA3- and CA1-FDSs, drugs were bath applied to slices if baseline recording of HTC-Waves was stable over $20 \mathrm{~min}$ (Figure 3A).

With the exception of fluvoxamine $(10 \mu \mathrm{M})$ and venlafaxine (1 and $10 \mu \mathrm{M}$ ), the ADs did not affect DG-FDSs (Figures 3A,B (left panels)). In contrast, at concentrations in the low micromolar range, all $\mathrm{ADs}$ led in the same experiments to an enhancement of CA1-FDSs (Figures 3A,B (black bars in right panels)). Interestingly, only the data obtained for fluoxetine, 

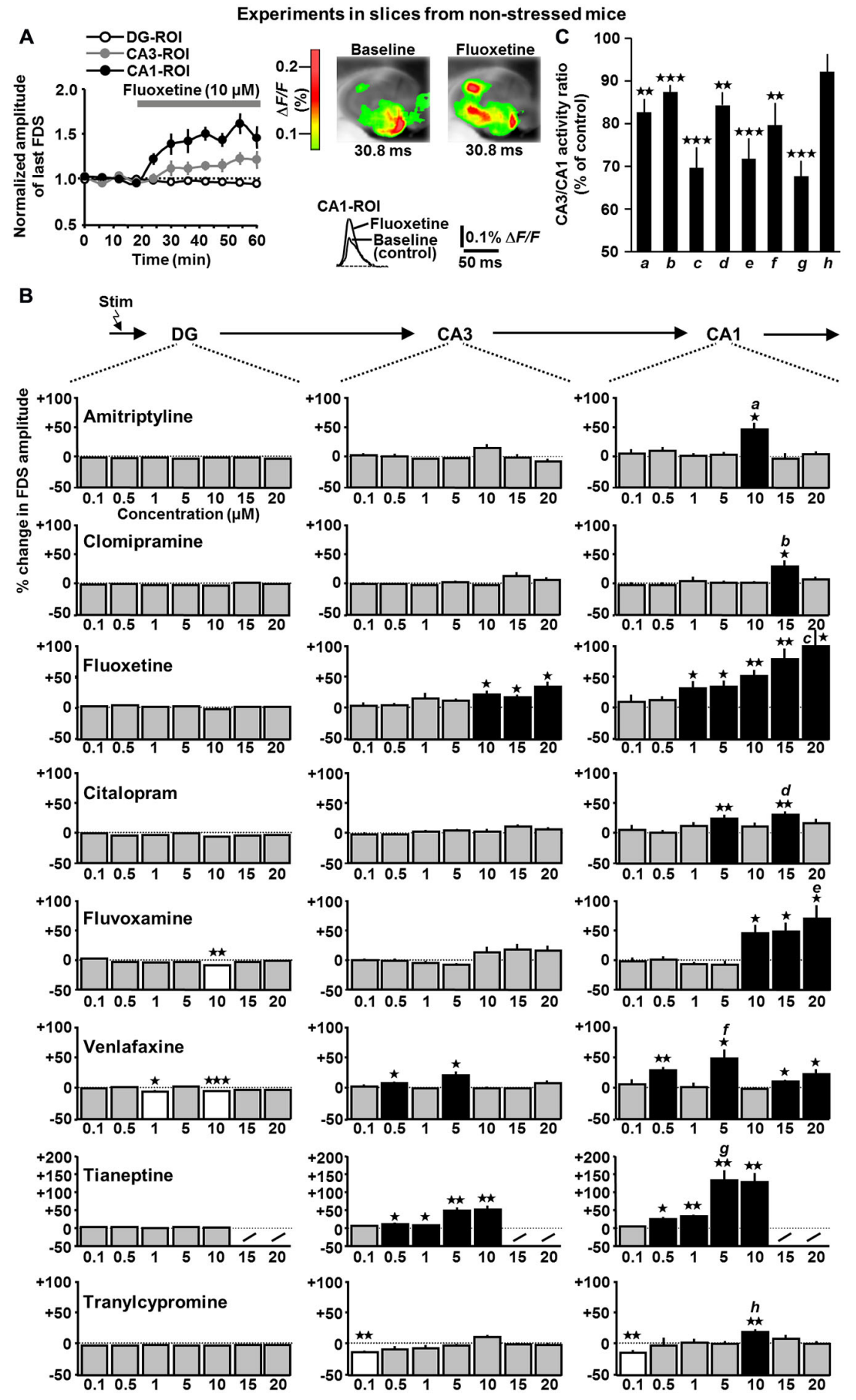

FIGURE 3 | Time course of fluoxetine effects (A) and concentration-dependent impact of amitriptyline, clomipramine, fluoxetine, citalopram, fluvoxamine, venlafaxine, tianeptine, and tranylcypromine (B) on HTC-Waves in slices from non-stressed mice. (A) As done with all other drugs probed, fluoxetine $(10 \mu \mathrm{M})$ was bath applied to slices $(n=7)$ if baseline recording of HTC-Waves was stable over 20 min. Subsequent recording time was always 40 min. Data were normalized to mean baseline FDS amplitude values. (B) For statistical analysis, one-way ANOVAs were applied to the DG-, CA3-, and CA1-FDS data sets (\% change in FDS amplitude, minutes 42-60 vs. minutes 0-18 of VSDI recording (A)) covering the whole range of concentrations probed. Due to its very strong effects at 5 and $10 \mu \mathrm{M}$, tianeptine was no longer tested at 15 and $20 \mu \mathrm{M}$. DG: fluvoxamine: $F_{(6,50)}=4.2, p=0.002 ; 10 \mu \mathrm{M}, t_{(7)}=-5.3, p=0.001 ;$ venlafaxine: $F_{(6,52)}=2.7, p=0.021 ; 1 \mu \mathrm{M}, t_{(8)}=-3.1, p=0.014 ; 10 \mu \mathrm{M}, t_{(8)}=-9.5, p<0.001 ;$ CA3: fluoxetine: $F_{(6.47)}=2.5, p=0.033 ; 10 \mu \mathrm{M}, t_{(6)}=3.5, p=0.013 ; 15 \mu \mathrm{M}$, $t_{(6)}=3.4, p=0.013 ; 20 \mu \mathrm{M}, t_{(7)}=3.4, p=0.011$; venlafaxine: $F_{(6,52)}=4.2, p=0.002 ; 0.5 \mu \mathrm{M}, t_{(9)}=2.6, p=0.027 ; 5 \mu \mathrm{M}, t_{(7)}=3.0, p=0.019 ;$ tianeptine: $F_{(4,30)}=8.8, p<0.001 ; 0.5 \mu \mathrm{M}, t_{(6)}=2.8, p=0.031 ; 1 \mu \mathrm{M}, t_{(6)}=2.6, p=0.043 ; 5 \mu \mathrm{M}, t_{(6)}=4.0, p=0.007 ; 10 \mu \mathrm{M}, t_{(6)}=4.4, p=0.005$; 


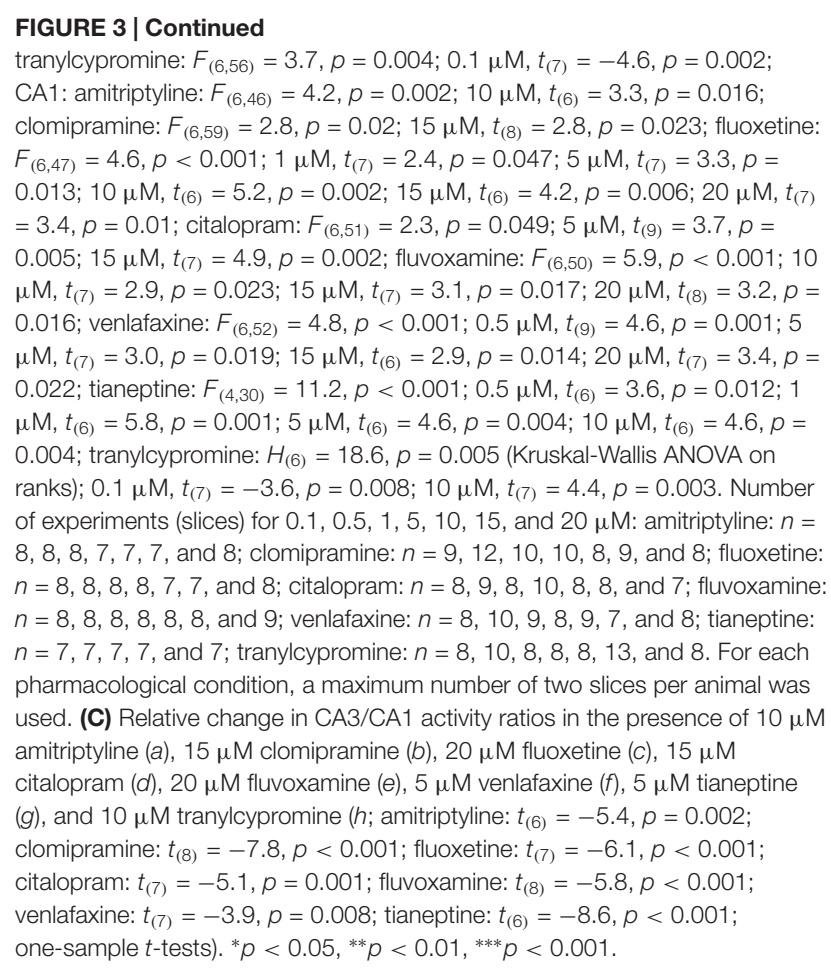

fluvoxamine, and tianeptine are reconcilable with a classical dose-response relationship. Fluoxetine (10, 15, and $20 \mu \mathrm{M})$, venlafaxine $(0.5$ and $5 \mu \mathrm{M})$, and tianeptine $(0.5,1,5$, and 10 $\mu \mathrm{M}$ ) additionally increased CA3-FDSs (Figures 3A,B (black bars in middle panels)). Another finding was that tranylcypromine $(0.1 \mu \mathrm{M})$ decreased CA3- and CA1-FDSs (Figure 3B). With the exception of tranylcypromine, all ADs reduced the CA3/CA1 activity ratio (Figure $3 \mathrm{C}$ ).

Taken together, at concentrations in the low micromolar range (but not at $0.1 \mu \mathrm{M}$ and mostly also not at $0.5 \mu \mathrm{M}$ ), all ADs caused an amplification of HTC-Waves in the CA1 output subfield of the hippocampus. At some of the effective doses, fluoxetine, venlafaxine, and tianeptine also provoked an enhancement of CA3 activity. A close inspection of the data depicted in Figure 3B suggests that the latter effect, although lacking statistical significance, likewise occurred with the other ADs.

\section{Lithium, BDNF, and Ketamine Exert Enhancing Effects on HTC-Waves}

We further tested whether other neuroactive factors, which do not belong to the group of classical ADs, but exhibit antidepressant-like actions, exert enhancing effects on HTCWaves. We examined potential effects of the mood stabilizer lithium (Moore et al., 2002), the neurotrophin BDNF (Shirayama et al., 2002), and the fast-acting "antidepressant" ketamine (Autry et al., 2011) on the activity propagations. In fact, without changing DG-FDSs, lithium (0.5 and $1 \mathrm{mM}$; Moore et al., 2002), BDNF (0.4 nM; Kang and Schuman, 1995), and ketamine (20 $\mathrm{MM}$; Autry et al., 2011) increased CA1-FDSs. BDNF and ketamine additionally caused an enhancement of CA3-FDSs (Figure 4A). As observed for most of the ADs, all three compounds reduced the CA3/CA1 activity ratio (Figure $4 \mathrm{~B}$ ).

\section{Haloperidol and Diazepam Weaken HTC-Waves}

The findings described above led us to hypothesize that, in comparison to other psychiatric drugs (e.g., antipsychotics and anxiolytics), an enhancing influence on HTC-Waves might be a characteristic feature of ADs. On this account, we next performed experiments with the classical antipsychotic haloperidol and the widely used anxiolytic diazepam (Benkert and Hippius, 2012). In line with our hypothesis, haloperidol decreased DG-, CA3-, and CA1-FDSs in a concentration-dependent manner. Consistent with its potentiating effect on $\mathrm{GABA}_{\mathrm{A}}$ receptor function and our previous demonstration that HTC-Waves are confined in their strength by GABAergic neurotransmission (Stepan et al., 2012), also diazepam (0.1 and $1 \mu \mathrm{M}$; Rupprecht et al., 2009) weakened the activity propagations (Figure 4C). In contrast to ADs, lithium, BDNF, and ketamine, haloperidol and diazepam increased the CA3/CA1 activity ratio (Figure 4B).

\section{Fluoxetine Exerts Enhancing Effects on HTC-Waves in Slices from Stressed Mice}

Next, we addressed the question of whether ADs also exert enhancing effects on HTC-Waves in slices obtained from chronically stressed mice. Hereunto, we investigated the impact of fluoxetine $(10 \mu \mathrm{M})$ on the activity propagations. We chose fluoxetine since it represents the classical $\mathrm{AD}$, which showed the most pronounced effects on HTC-Waves (Figure 3B). Indeed, as observed in naive slices (for statistics see Figure 3), fluoxetine increased CA3- and CA1-FDSs in slices from stressed animals (Figure 5 (middle bars)).

\section{The TrkB Receptor Antagonist ANA-12 Prevents Fluoxetine Effects on HTC-Waves}

There is substantial evidence that activation of the BDNF receptor TrkB is involved in the mechanisms of action of ADs (Castrén and Rantamäki, 2010). Our finding that BDNF, like ADs, increases FDSs in CA regions led us to test a possible contribution of TrkB activation to the AD effects on HTCWaves. Hereunto, we preincubated slices for at least $1 \mathrm{~h}$ with the TrkB antagonist ANA-12 (10 $\mu \mathrm{M}$; Longo and Massa, 2013). Afterwards, we again investigated the impact of fluoxetine (10 $\mu \mathrm{M}$, for rationale see above) on HTC-Waves. As shown in Figure 5 (right bars), ANA-12 prevented the fluoxetine-induced amplification of CA1- and CA3-FDSs.

\section{DISCUSSION}

In the present study, we show that eight clinically used ADs (although belonging to different chemical/functional classes) cause an amplification of HTC-Waves in CA regions (invariably in the CA1 subfield and also partly in area CA3). However, these effects only occurred at concentrations in the low micromolar range, with the exception of venlafaxine and tianeptine, which 


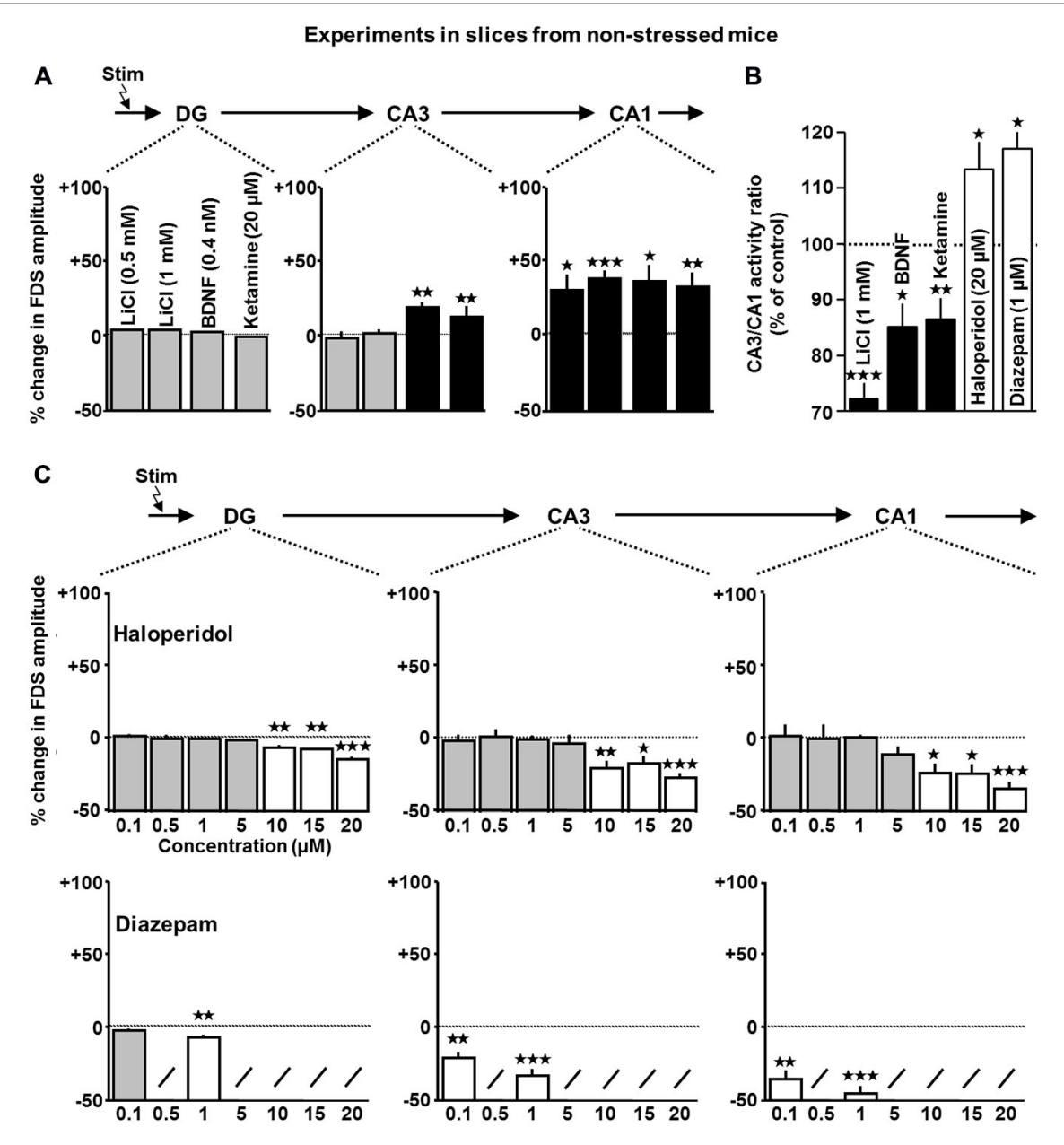

FIGURE 4 | Impact of lithium, BDNF, ketamine, haloperidol, and diazepam on HTC-Waves in slices from non-stressed mice. (A) For statistical analysis, one-sample $t$-tests were used (lithium ( $n=7$ slices for 0.5 and $1 \mathrm{mM}$ ): CA1: $0.5 \mathrm{mM}, t_{(6)}=2.5, p=0.045 ; 1 \mathrm{mM}, t_{(6)}=7.5, p<0.001$; BDNF $(n=8$ slices): CA3: $t_{(7)}=3.6, p=0.008 ;$ CA1: $t_{(7)}=3.4, p=0.011$; ketamine $\left(n=8\right.$ slices): CA3: $t_{(7)}=4.9, p=0.002 ;$ CA1: $\left.t_{(7)}=4.2, p=0.004\right)$. (B) Relative change in CA3/CA1 activity ratios in the presence of $1 \mathrm{mM}$ lithium (a), $0.4 \mathrm{nM}$ BDNF (b), $20 \mu \mathrm{M}$ ketamine (c), $20 \mu \mathrm{M}$ haloperidol (d), and $1 \mu \mathrm{M}$ diazepam (e; lithium: $t_{(6)}=-9.7, p<$ 0.001; BDNF: $t_{(7)}=-3.5, p=0.01$; ketamine: $t_{(7)}=-3.6, p=0.009$; haloperidol: $t_{(5)}=2.7, p=0.035$; diazepam: $t_{(5)}=3.3, p=0.029$; one-sample $t$-tests). (C) Statistical evaluation of haloperidol effects was performed as done for ADs. For diazepam effects, the one-sample $t$-test was used (haloperidol: DG: $F_{(6,43)}=17.8$, $p<0.001 ; 10 \mu \mathrm{M}, t_{(7)}=-4.3, p=0.003 ; 15 \mu \mathrm{M}, t_{(5)}=-4.8, p=0.005 ; 20 \mu \mathrm{M}, t_{(5)}=-10.1, p<0.001 ; \mathrm{CA3}: F_{(6,43)}=4.3, p=0.002 ; 10 \mu \mathrm{M}, t_{(7)}=-3.7, p=$ $0.007 ; 15 \mu \mathrm{M}, t_{(5)}=-3.0, p=0.028 ; 20 \mu \mathrm{M}, t_{(5)}=-8.7, p<0.001 ;$ CA1: $F_{(6,43)}=3.6, p=0.005 ; 10 \mu \mathrm{M}, t_{(7)}=-3.5, p=0.01 ; 15 \mu \mathrm{M}, t_{(5)}=-3.5, p=0.017 ; 20$ $\mu \mathrm{M}, t_{(5)}=-9.0, p<0.001$; number of experiments (slices) for $0.1,0.5,1,5,10,15$, and $20 \mu \mathrm{M}: n=8,8,6,8,8,6$, and 6 ; diazepam ( $n=7$ slices for $0.1 \mu \mathrm{M}$ and $n=6$ slices for $1 \mu \mathrm{M})$ : DG: $1 \mu \mathrm{M}, t_{(5)}=-4.1, p=0.009 ;$ CA3: $0.1 \mu \mathrm{M}, t_{(6)}=-4.9, p=0.003 ; 1 \mu \mathrm{M}, t_{(5)}=-7.8, p<0.001 ; \mathrm{CA} 1: 0.1 \mu \mathrm{M}, t_{(6)}=-5.7, p=0.001 ; 1$ $\left.\mu \mathrm{M}, t_{(5)}=-8.4, p<0.001\right)$. For each pharmacological condition, a maximum number of two slices per animal was used. ${ }^{*} p<0.05,{ }^{* *} p<0.01,{ }^{* * *} p<0.001$.

additionally slightly boosted the activity propagations at $0.5 \mu \mathrm{M}$. Low micromolar brain concentrations of ADs are clinically relevant (Strauss et al., 1997; Bolo et al., 2000; Henry et al., 2005; Méndez et al., 2012), but considerably higher than those required for monoamine reuptake inhibition $\left(\mathrm{IC}_{50}\right.$ values for serotonin reuptake inhibition by amitriptyline, clomipramine, fluoxetine, citalopram, and fluvoxamine (in $\mathrm{nM}$ ): 39, 1.5, 6.8, 1.8, and 3.8, respectively (Hyttel, 1993)). In combination with our findings that also the serotonin reuptake enhancer tianeptine and BDNF increase CA3- and CA1-FDSs and that the TrkB receptor antagonist ANA-12 prevents the effects of fluoxetine $(10 \mu \mathrm{M})$, it is thus possible that the AD effects observed do not depend on enhanced monoaminergic neurotransmission, but, at least in part, on increased TrkB signaling. Consistently, activation of $\operatorname{TrkB}$ is involved in $\mathrm{AD}$ actions and also lithium and ketamine, which likewise amplified HTC-Waves, enforce TrkB signaling (Castrén and Rantamäki, 2010; Autry et al., 2011).

Notably, only the data obtained for fluoxetine, fluvoxamine, and tianeptine are reconcilable with a classical dose-response relationship. The lack of such a relationship observed for the other five $\mathrm{ADs}$ and the fact that three compounds produced a boosting effect on HTC-Waves merely at one of the concentrations probed presumably rely on the partly intricate and heterogeneous pharmacological properties of ADs and the high structural and functional complexity of the 
HTC network (i.e., trisynaptic interconnections and appendant excitatory/inhibitory microcircuits; Andersen et al., 2007). This might also be the reason why fluvoxamine $(10 \mu \mathrm{M})$, venlafaxine (1 and $10 \mu \mathrm{M})$, and tranylcypromine $(0.1 \mu \mathrm{M})$ decreased DGor CA3- and CA1-FDSs (Figure 3B). For instance, beside its enhancing action on monoaminergic neurotransmission, amitriptyline inhibits nicotinic acetylcholine receptors, usedependently blocks voltage-gated sodium channels, and activates TrkB receptors with different potencies (Schofield et al., 1981; Huang et al., 2006; Jang et al., 2009). Similar (but not identical) pharmacological profiles have been described for some of the other ADs tested, whereas those of venlafaxine, tianeptine, and tranylcypromine are considerably different (Huang et al., 2006; Castrén and Rantamäki, 2010; McEwen et al., 2010; Benkert and Hippius, 2012). It is thus conceivable that a particular AD (at concentration A) exerts an enhancing effect on HTC-Waves, while, at a higher concentration $\mathrm{B}$, this effect is less pronounced or absent (cf., Figure 3B (upper right panel)). Another AD in turn might show a varying effect pattern. Therefore, we deem it extremely challenging, if not impossible, to accurately predict AD actions on large/meso-scale neuronal network dynamics from known effects at the molecular/cellular level, given that these effects presumably have not been completely elucidated until now.

We yield evidence that, in comparison to antipsychotics and anxiolytics, enhancing effects on HTC-Waves are a characteristic feature of $\mathrm{ADs}$ and other neuroactive factors, which show antidepressant-like actions. This evidence is given by our findings that lithium, BDNF, and ketamine also cause an amplification of HTC-Waves, whereas haloperidol and diazepam weaken the activity propagations. The data obtained for haloperidol are noteworthy, since an impaired hippocampal

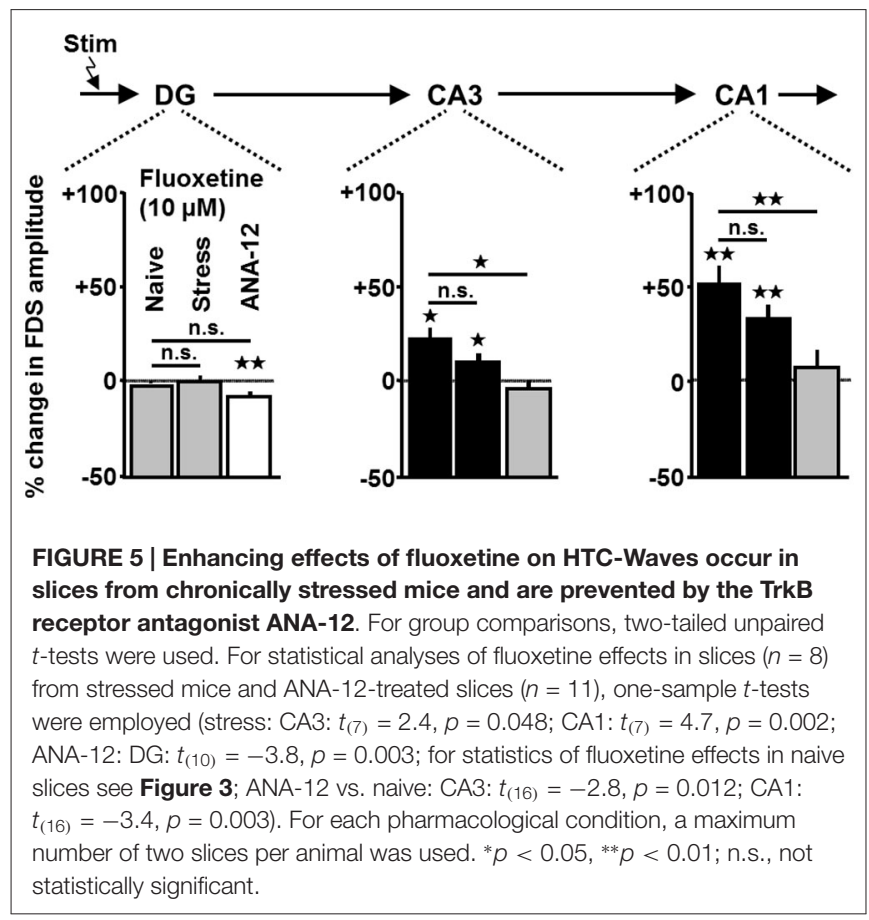

gating of sensory information flow from the entorhinal cortex to downstream brain structures has been implicated in the pathophysiology of schizophrenia (e.g., Javanbakht, 2006).

All slice experiments in the present study were performed in the presence of BIM $(0.6 \mu \mathrm{M})$. We used the competitive $\mathrm{GABA}_{\mathrm{A}}$ receptor antagonist BIM at this weakly blocking dose (Stepan et al., 2012) for the following reasons. First, compared to the relatively short axons of most hippocampal GABAergic interneurons (Andersen et al., 2007), projections from excitatory neurons are cut to a greater extent during preparation of slices. This probably leads to an artificial enhancement of inhibition in large-scale hippocampal circuits in vitro (e.g., Iijima et al., 1996). And second, the voltage-sensitive dye Di-4-ANEPPS possibly slightly potentiated $\mathrm{GABA}_{\mathrm{A}}$ receptor function (Mennerick et al., 2010). Hence, BIM most likely gave rise to more physiological conditions, rather than qualitatively artificial pharmacological effects. Consistently, HTC-Waves were weakened by the $\mathrm{GABA}_{\mathrm{A}}$ receptor potentiator diazepam.

We revealed that chronic social defeat stress causes markedly attenuated HTC-Waves, providing direct evidence that chronic stress can depress neuronal signal flow through the HTC. Furthermore, this finding suggests that the enhancing effects of ADs on HTC-Waves are therapeutically relevant. Importantly, fluoxetine also boosted CA3- and CA1-FDSs in slices from stressed mice and the stress paradigm used causes impaired hippocampus-dependent cognitive abilities, representing a typical symptom of depression and other stress-related psychiatric disorders (Wang et al., 2011; Wagner et al., 2012). But how could the $\mathrm{AD}$ effects observed contribute to an alleviation of this symptom? It is tempting to speculate that a strengthening of activity-dependent neuroplastic processes plays a critical role (Castrén, 2005). For instance, NMDA receptor-dependent long-term potentiation (LTP) at CA3-CA1 synapses (CA1 LTP), which is widely accepted to be crucial for some hippocampusdependent cognitive abilities (Burgess et al., 2002), can be induced by HTC-Waves (Stepan et al., 2012) and possesses the properties of "cooperativity" and "associativity" (Bliss and Collingridge, 1993). Therefore, weakened activity propagations through the HTC should be less effective in the induction of CA1 LTP, whereas enhancing AD effects on them probably counteract this impairment. In this context, it is important to mention that a recent publication likewise points to an enhancement of CA1 activity by ADs. This study specifically investigated AD actions on CA1 GABAergic neurotransmission (Méndez et al., 2012). The effects observed by Méndez et al. (2012) did not depend on enhanced monoaminergic neurotransmission and required $\mathrm{AD}$ concentrations in the low micromolar range. Notably, investigations in humans revealed that fluoxetine and fluvoxamine reach low micromolar concentrations (10-15 $\mu \mathrm{M}$; cf., Figure 3B) in the brain after continuous drug administration for 30 days or longer, matching the time point at which $\mathrm{AD}$ therapy usually starts to be effective. One or two days after start of treatment, the brain concentration is much lower (low to intermediate nanomolar range; Strauss et al., 1997; Bolo et al., 2000). It is thus conceivable that the onset of beneficial $\mathrm{AD}$ actions in part reflects acute drug effects on brain physiology, as it is the case for ketamine. These remarks 
justify the investigation of fast modulatory actions of ADs on neurophysiological processes, as done here and in many previous studies (e.g., Schofield et al., 1981; Jang et al., 2009; Méndez et al., 2012). However, further work using chronic $\mathrm{AD}$ administration is needed to corroborate the potential therapeutic relevance of the enhancing AD effects on HTCWaves.

As already mentioned in the introduction section, also Airan et al. (2007) employed VSDI in brain slices to examine chronic stress and $\mathrm{AD}$ effects on hippocampal circuit dynamics. However, due to the partly big differences in the experimental design (see Introduction), a direct comparison with our findings is hardly achievable. Yet, if the measure "activity percolation" through area CA1 should reflect activity strength, our results do not fit to those of Airan et al. (2007). This is because our data point to an impaired neurotransmission in area CA1 of stressed mice and an opposite scenario in the presence of ADs (Figures 2G, 3C). In contrast, Airan et al. reported an increased "activity percolation" in slices from stressed rats, which is counterintuitive with regard to the well-known detrimental actions of chronic stress on the structural and functional integrity of the hippocampus, and observed an antipodal effect if animals were treated with fluoxetine or imipramine. However, one has to consider that Airan and colleagues induced CA1 activity by single-pulse stimulation of the pyramidal cell layer and the ADs were most likely washed

\section{REFERENCES}

Airan, R. D., Meltzer, L. A., Roy, M., Gong, Y., Chen, H., and Deisseroth, K. (2007). High-speed imaging reveals neurophysiological links to behavior in an animal model of depression. Science 317, 819-823. doi: 10.1126/science.1144400

Andersen, P., Morris, R., Amaral, D., Bliss, T., and O'Keefe, J. (2007). The Hippocampus Book. New York, NY: Oxford University Press.

Autry, A. E., Adachi, M., Nosyreva, E., Na, E. S., Los, M. F., Cheng, P. F., et al. (2011). NMDA receptor blockade at rest triggers rapid behavioural antidepressant responses. Nature 475, 91-95. doi: 10.1038/nature10130

Benkert, O., and Hippius, H. (2012). Kompendium der Psychiatrischen Pharmakotherapie. Berlin, Germany: Springer.

Bischofberger, J., Engel, D., Li, L., Geiger, J. R. P., and Jonas, P. (2006). Patchclamp recording from mossy fiber terminals in hippocampal slices. Nat. Protoc. 1, 2075-2081. doi: 10.1038/nprot.2006.312

Bliss, T. V., and Collingridge, G. L. (1993). A synaptic model of memory: long-term potentiation in the hippocampus. Nature 361, 31-39. doi: 10.1038/361031a0

Bolo, N. R., Hodé, Y., Nédélec, J. F., Lainé, E., Wagner, G., and Macher, J. P. (2000). Brain pharmacokinetics and tissue distribution in vivo of fluvoxamine and fluoxetine by fluorine magnetic resonance spectroscopy. Neuropsychopharmacology $\quad 23, \quad 428-438$. doi: $10.1016 / \mathrm{s} 0893-133 x(00)$ 00116-0

Burgalossi, A., Herfst, L., von Heimendahl, M., Förste, H., Haskic, K., Schmidt, M., et al. (2011). Microcircuits of functionally identified neurons in the rat medial entorhinal cortex. Neuron 70, 773-786. doi: 10.1016/j.neuron.2011.04.003

Burgess, N., Maguire, E. A., and O'Keefe, J. (2002). The human hippocampus and spatial and episodic memory. Neuron 35, 625-641. doi: 10.1016/s08966273(02)00830-9

Castrén, E. (2005). Is mood chemistry? Nat. Rev. Neurosci. 6, 241-246. doi: 10. $1038 /$ nrn 1629

Castrén, E., and Rantamäki, T. (2010). The role of BDNF and its receptors in depression and antidepressant drug action: reactivation of developmental plasticity. Dev. Neurobiol. 70, 289-297. doi: 10.1002/dneu.20758 out from their slice preparations. CA1 activity in our study resulted from burst firing of CA3 pyramidal neurons, which is a typical discharge pattern of these cells (Stepan et al., 2012). An identical comparison for the DG data cannot be drawn, since we adjusted DG activity in slices from stressed mice to predefined values.

In summary, we provide evidence for shared modulatory effects of ADs on HTC dynamics that counteract stress-induced impairment of hippocampal network function. These effects, which might be valuable for the screening for novel ADs, point to a circuit-level mechanism of ADs in the entorhinalhippocampal system to alleviate cognitive dysfunctions often observed with depression and other stress-related psychiatric conditions. However, these effects cannot depend on enhanced neurogenesis (Hill et al., 2015). Hence, our work highlights the importance of circuit-centered approaches, complementary to investigations at the molecular/cellular level and brain imaging studies, in order to achieve a comprehensive understanding of how environmental risk factors can translate into brain disease states and how psychiatric drugs mediate their beneficial actions.

\section{ACKNOWLEDGMENTS}

We thank Carsten T. Wotjak, Evan D. Paul, and Julien Dine for valuable comments on the manuscript.

de Kloet, E. R., Joëls, M., and Holsboer, F. (2005). Stress and the brain: from adaptation to disease. Nat. Rev. Neurosci. 6, 463-475. doi: 10.1038/nrn1683

Dickson, C. T., Magistretti, J., Shalinsky, M., Hamam, B., and Alonso, A. (2000). Oscillatory activity in entorhinal neurons and circuits. Mechanisms and function. Ann. N Y Acad. Sci. 911, 127-150. doi: 10.1111/j.1749-6632.2000. tb06723.x

Henry, M. E., Schmidt, M. E., Hennen, J., Villafuerte, R. A., Butman, M. L., Tran, P., et al. (2005). A comparison of brain and serum pharmacokinetics of R-fluoxetine and racemic fluoxetine: a 19-F MRS study. Neuropsychopharmacology 30, 1576-1583. doi: 10.1038/sj.npp. 1300749

Hill, A. S., Sahay, A., and Hen, R. (2015). Increasing adult hippocampal neurogenesis is sufficient to reduce anxiety and depression-like behaviors. Neuropsychopharmacology 40, 2368-2378. doi: 10.1038/npp.2015.85

Huang, C. J., Harootunian, A., Maher, M. P., Quan, C., Raj, C. D., McCormack, K., et al. (2006). Characterization of voltage-gated sodium-channel blockers by electrical stimulation and fluorescence detection of membrane potential. Nat. Biotechnol. 24, 439-446. doi: 10.1038/nbt1194

Hyttel, J. (1993). Comparative pharmacology of selective serotonin reuptake inhibitors (SSRIs). Nord. J. Psychiatry 47, 5-12. doi: 10. 3109/08039489309104119

Iijima, T., Witter, M. P., Ichikawa, M., Tominaga, T., Kajiwara, R., and Matsumoto, G. (1996). Entorhinal-hippocampal interactions revealed by real-time imaging. Science 272, 1176-1179. doi: 10.1126/science.272.5265. 1176

Jang, S. W., Liu, X., Chan, C. B., Weinshenker, D., Hall, R. A., Xiao, G., et al. (2009). Amitriptyline is a TrkA and TrkB receptor agonist that promotes TrkA/TrkB heterodimerization and has potent neurotrophic activity. Chem. Biol. 16, 644-656. doi: 10.1016/j.chembiol.2009.05.010

Javanbakht, A. (2006). Sensory gating deficits, pattern completion and disturbed fronto-limbic balance, a model for description of hallucinations and delusions in schizophrenia. Med. Hypotheses 67, 1173-1184. doi: 10.1016/j.mehy.2006. 03.054 
Kang, H., and Schuman, E. M. (1995). Long-lasting neurotrophin-induced enhancement of synaptic transmission in the adult hippocampus. Science 267, 1658-1662. doi: 10.1126/science.7886457

Karayiorgou, M., Flint, J., Gogos, J. A., and Malenka, R. C. (2012). The best of times, the worst of times for psychiatric disease. Nat. Neurosci. 15, 811-812. doi: 10.1038/nn.3115

Krishnan, V., and Nestler, E. J. (2008). The molecular neurobiology of depression. Nature 455, 894-902. doi: 10.1038/nature07455

Longo, F. M., and Massa, S. M. (2013). Small-molecule modulation of neurotrophin receptors: a strategy for the treatment of neurological disease. Nat. Rev. Drug Discov. 12, 507-525. doi: 10.1038/nrd4024

Maggio, N., and Segal, M. (2007). Striking variations in corticosteroid modulation of long-term potentiation along the septotemporal axis of the hippocampus. J. Neurosci. 27, 5757-5765. doi: 10.1523/jneurosci.0155-07.2007

McEwen, B. S., Chattarji, S., Diamond, D. M., Jay, T. M., Reagan, L. P., Svenningsson, P., et al. (2010). The neurobiological properties of tianeptine (Stablon): from monoamine hypothesis to glutamatergic modulation. Mol. Psychiatry 15, 237-249. doi: 10.1038/mp.2009.80

Méndez, P., Pazienti, A., Szabó, G., and Bacci, A. (2012). Direct alteration of a specific inhibitory circuit of the hippocampus by antidepressants. J. Neurosci. 32, 16616-16628. doi: 10.1523/JNEUROSCI.1720-12.2012

Mennerick, S., Chisari, M., Shu, H. J., Taylor, A., Vasek, M., Eisenman, L. N., et al. (2010). Diverse voltage-sensitive dyes modulate $\mathrm{GABA}_{\mathrm{A}}$ receptor function. J. Neurosci. 30, 2871-2879. doi: 10.1523/JNEUROSCI.5607-09. 2010

Mizuseki, K., Sirota, A., Pastalkova, E., and Buzsáki, G. (2009). Theta oscillations provide temporal windows for local circuit computation in the entorhinalhippocampal loop. Neuron 64, 267-280. doi: 10.1016/j.neuron.2009.08.037

Monteggia, L. M., Malenka, R. C., and Deisseroth, K. (2014). Depression: the best way forward. Nature 515, 200-201. doi: 10.1038/515200a

Moore, C. M., Demopulos, C. M., Henry, M. E., Steingard, R. J., Zamvil, L., Katic, A., et al. (2002). Brain-to-serum lithium ratio and age: an in vivo magnetic resonance spectroscopy study. Am. J. Psychiatry 159, 1240-1242. doi: 10. 1176/appi.ajp.159.7.1240

Popoli, M., Yan, Z., McEwen, B. S., and Sanacora, G. (2012). The stressed synapse: the impact of stress and glucocorticoids on glutamate transmission. Nat. Rev. Neurosci. 13, 22-37. doi: 10.1038/nrn3138

Quilichini, P., Sirota, A., and Buzsáki, G. (2010). Intrinsic circuit organization and theta-gamma oscillation dynamics in the entorhinal cortex of the rat. J. Neurosci. 30, 11128-11142. doi: 10.1523/JNEUROSCI.1327-10. 2010

Rupprecht, R., Rammes, G., Eser, D., Baghai, T. C., Schüle, C., Nothdurfter, C., et al. (2009). Translocator protein $(18 \mathrm{kD})$ as target for anxiolytics without benzodiazepine-like side effects. Science 325, 490-493. doi: 10.1126/science. 1175055
Schofield, G. G., Witkop, B., Warnick, J. E., and Albuquerque, E. X. (1981). Differentiation of the open and closed states of the ionic channels of nicotinic acetylcholine receptors by tricyclic antidepressants. Proc. Natl. Acad. Sci. U S A 78, 5240-5244. doi: 10.1073/pnas.78.8.5240

Shirayama, Y., Chen, A. C., Nakagawa, S., Russell, D. S., and Duman, R. S. (2002). Brain-derived neurotrophic factor produces antidepressant effects in behavioral models of depression. J. Neurosci. 22, 3251-3261.

Stepan, J., Dine, J., and Eder, M. (2015). Functional optical probing of the hippocampal trisynaptic circuit in vitro: network dynamics, filter properties and polysynaptic induction of CA1 LTP. Front. Neurosci. 9:160. doi: 10 3389/fnins.2015.00160

Stepan, J., Dine, J., Fenzl, T., Polta, S. A., von Wolff, G., Wotjak, C. T., et al. (2012). Entorhinal theta-frequency input to the dentate gyrus trisynaptically evokes hippocampal CA1 LTP. Front. Neural Circuits 6:64. doi: 10.3389/fncir.2012. 00064

Strauss, W. L., Layton, M. E., Hayes, C. E., and Dager, S. R. (1997). 19F magnetic resonance spectroscopy investigation in vivo of acute and steady-state brain fluvoxamine levels in obsessive-compulsive disorder. Am. J. Psychiatry 154, 516-522. doi: 10.1176/ajp.154.4.516

von Wolff, G., Avrabos, C., Stepan, J., Wurst, W., Deussing, J. M., Holsboer, F., et al. (2011). Voltage-sensitive dye imaging demonstrates an enhancing effect of corticotropin-releasing hormone on neuronal activity propagation through the hippocampal formation. J. Psychiatr. Res. 45, 256-261. doi: 10.1016/j.jpsychires. 2010.06.007

Wagner, K. V., Marinescu, D., Hartmann, J., Wang, X. D., Labermaier, C., Scharf, S. H., et al. (2012). Differences in FKBP51 regulation following chronic social defeat stress correlate with individual stress sensitivity: influence of paroxetine treatment. Neuropsychopharmacology 37, 2797-2808. doi: 10.1038/npp. 2012.150

Wang, X. D., Chen, Y., Wolf, M., Wagner, K. V., Liebl, C., Scharf, S. H., et al. (2011). Forebrain CRHR1 deficiency attenuates chronic stress-induced cognitive deficits and dendritic remodeling. Neurobiol. Dis. 42, 300-310. doi: 10.1016/j.nbd.2011.01.020

Conflict of Interest Statement: The authors declare that the research was conducted in the absence of any commercial or financial relationships that could be construed as a potential conflict of interest.

Copyright (c) 2015 Stepan, Hladky, Uribe, Holsboer, Schmidt and Eder. This is an open-access article distributed under the terms of the Creative Commons Attribution License (CC BY). The use, distribution and reproduction in other forums is permitted, provided the original author(s) or licensor are credited and that the original publication in this journal is cited, in accordance with accepted academic practice. No use, distribution or reproduction is permitted which does not comply with these terms. 